\title{
Modified Hanging Maneuver Using the Goldfinger Dissector in Laparoscopic Right and Left Hepatectomy
}

\author{
Roberto I. Troisi Roberto Montalti \\ Department of General and Hepato-Biliary Surgery, Liver Transplantation Service, Ghent University Hospital and \\ Medical School, Ghent, Belgium
}

\section{Key Words}

Modified liver hanging maneuver - Goldfinger dissector .

Laparoscopic liver resection

\section{Abstract}

Background: The liver hanging maneuver is a useful technique to transect the liver parenchyma in an open approach while lifting it with a tape passed between the anterior surface of the inferior vena cava and the liver parenchyma. The hanging maneuver during laparoscopic liver resection is not routinely utilized. Materials and Methods: We describe the technique and results of a modified hanging maneuver using the Goldfinger dissector in 13 right and 5 left hepatectomies. This technique allowed us to dissect the space between right and middle hepatic veins; to create the inferosuperior arcade through the space between the anterior surface of the vena cava and the posterior surface of the liver; to lift the cutting area of the liver from the posterior to the anterior direction; to indicate the parenchymal transection line. Results: The median resection time and blood loss were $96 \mathrm{~min}$ and $220 \mathrm{ml}$ respectively. No conversion was recorded. Two complications (11\%) occurred: a biliary fistula and a respiratory distress syndrome, spontaneously resolved. Con- clusions: The modified hanging maneuver for laparoscopic right and left hepatectomy based on the use of the Goldfinger dissector is safe, reproducible, and can facilitate liver dissection during major hepatectomy.

Copyright $\odot 2012$ S. Karger AG, Basel

\section{Background}

Laparoscopic liver resection (LLR) is nowadays an increasingly used technique worldwide, documented in more than 355 original published papers [1]. Although LLR initially favored benign lesions, the first indications are actually primary and secondary liver tumors, the oncological results being comparable to those obtained with a standard approach $[2,3]$. Moreover, with the progressive increase in the surgeons' experience, any kind of liver resection that does not require a vascular or biliary reconstruction can be performed laparoscopically.

The liver hanging maneuver, already described by Belghiti et al. [4] in 2001, is a technique used to transect the liver parenchyma in an open approach in order to facilitate the anterior approach avoiding liver mobilization. This consists in a tape passed between the anterior surface

\section{KARGER}

Fax +4161306 1234

E-Mail karger@karger.com

www.karger.com
C 2012 S. Karger AG, Basel

0253-4886/12/0296-0463\$38.00/0

Accessible online at:

www.karger.com/dsu
Roberto I. Troisi, MD, PhD, FEBS

Department of General and Hepato-Biliary Surgery, Liver Transplantation Service, Ghent University Hospital Medical School

De Pintelaan 185, 2K12 IC, BE-9000 Ghent (Belgium)

E-Mail roberto.troisi@ugent.be 
of the inferior vena cava (IVC) and the liver parenchyma through the right (RHV) and middle hepatic veins (MHV) to lift up the liver and facilitate its division. The hanging maneuver is useful to guide the transection plane and facilitates the control of bleeding in the deeper parenchyma while protecting the anterior surface of the IVC [4]. This technique, however, is not routinely applied in LLR due to some intrinsic difficulty in hanging the liver with a tape.

Conversely, in the present paper, we describe the feasibility of a modified hanging maneuver for LLRs in right and left hepatectomy without the positioning of a tape.

\section{Materials and Methods}

Between May 2009 and December 2011, a total of 297 liver resections were done in 267 patients. Pure LLRs were performed in 130 (43.7\%) cases. Right or left LLR was indicated in 31 (23.8\%) of patients. In the last 18 major hepatectomies, we utilized a modified hanging maneuver using the Goldfinger ${ }^{\odot}$ (Blunt Dissector and Suture Retrieval System, Ethicon Endo Surgery, Johnson \& Johnson, New Brunswick, N.J., USA). The Goldfinger is an endoscopic dissector previously developed for laparoscopic bariatric surgery that helps to position the gastric banding around the stomach. Recently, it has also been reported to facilitate Pringle's maneuver and distal pancreatectomy [5-7] (fig. 1). The tip of the Goldfinger dissector can flex more than $90^{\circ}$ in the vertical axis, which is similar to the movement obtained by flexing a finger. It has multipositional flexibility, is malleable and provides precise articulation. Moreover, the tip is atraumatic and at the end has a chink for tape insertion, whereas the body is rigid enough to lift the liver. The Goldfinger has a one-handed, ergonomic operation, which enables precise dissection and controlled grasping. It is also disposable and ensures sterility and consistent performance [6].

\section{General Surgical Technique}

LLR was performed with the patient in the 30-degree antiTrendelenburg position and the surgeons between the patient's legs. An intermittent compression device was applied to the lower extremities to minimize the risk of deep venous thrombosis. For planned left hepatectomy, the patient is in the supine position, whereas for right hepatectomy, a pillow is placed behind the right hypochondrium.

Basically, 4-6 port sites ( $5 \mathrm{~mm}, 10 \mathrm{~mm}$ and 4 of $12 \mathrm{~mm}$ ) were inserted on the upper abdominal quadrant: the $12-\mathrm{mm}$ ports were placed to allow insertion of a 30-degree optical device and the linear stapler; the $10-\mathrm{mm}$ port was utilized for the surgical aspirator or harmonic scissors, and the 5-mm port was used mainly to allow irrigation and aspiration during surgery, and to hang the liver when necessary. Carbon dioxide pressure for pneumoperitoneum was kept aiming at $10-12 \mathrm{~mm} \mathrm{Hg}$ during hepatic parenchyma transection. Assessment of liver surface and surgical margins was done by intraoperative ultrasonography guidance (Aloka SSD 4000, Tokyo, Japan). Parenchyma division was performed using the surgical aspirator (CUSA, Excel Valleylab until 2009 and SonoSurg-Olympus to date). Hemostasis and biliostasis were done with bipolar coagulation and clips (Hem-o-lock clips, TFX Medical Ltd., RTP Durham, N.C., USA). Larger vascular/biliary structures were controlled by vascular staplers (EndoGIA Ethicon; EndoGia ${ }^{\mathrm{TM}}$ Tristaple $^{\mathrm{TM}}$ Technology Covidien). Finally, the specimen was extracted using a plastic bag through a Pfannenstiel incision or by partial opening of a previous midline abdominal scar.

\section{Modified Hanging Maneuver Technique}

Right Hepatectomy. Following ultrasound exploration, the falciform ligament is cut and the round ligament is retracted in a cephalad direction to expose the hilar region. After cholecystectomy, the main portal vein is mobilized up to the bifurcation. After dividing the right triangular and coronary ligaments, we gently dissect the upper space between the RHV and MHV with the Goldfinger (fig. 2a). The IVC and part of the right adrenal gland and retroperitoneum are dissected in a caudal to cephalad direction, working toward the hepatic veins to allow the liver to be lifted up with the Goldfinger. Small perforating vessels and accessory hepatic veins are controlled with bipolar coagulation or, in the case of larger vessels, by clips. The right hepatic artery and portal vein are isolated, clipped separately and divided. Liver transection starts with an inferior-superior approach following the demarcation of the ischemic line. When liver division is about half-way, we use the modified hanging maneuver for LLR, inserting the Goldfinger through the avascular route on the midline of the anterior surface of the infrahepatic portion of the IVC and lifting up the cutting area (fig. $2 \mathrm{~b}-\mathrm{d}$ ). We prefer to first dissect one third of the liver parenchyma to enhance the lifting and exposure effects of the Goldfinger when a part of the parenchyma is already divided. Unlike in open surgery where the dissection route is anterior to posterior, in LLR this route is inferior to superior, and more space is needed to achieve the ideal exposure and exploit the help offered by this method. As soon as the bile ducts have been identified, the stapler is used to divide and secure them. Then, by hanging the liver that is also a little bit pulled toward the surgical aspirator, the parenchyma division is completed, the RHV is exposed, lifted by the Goldfinger, and a second stapler is used to complete vascular parenchymal dissection.

Left Hepatectomy. The left hemiliver is mobilized by dividing the left triangular and coronary ligament identifying the ligamentum venosum. By meticulous blunt dissection, the left hepatic artery and portal vein are separated, secured by the Hem-olock clips, then divided. Dissection of the MHV and left hepatic vein (LHV) depends upon the need to include or not the MHV within the resection specimen. The Goldfinger is used to hang the left liver during dissection. Liver parenchyma is transected along the Cantlie line in a similar fashion to the right hepatectomy, following the ischemic line with the infero-superior approach until about half-way through the liver resection. Once the dissection has reached the liver hilum exposing the biliary ducts, a first stapler is fired to secure and divide it. The Goldfinger is then inserted below the parenchyma to lift the liver and then placed behind the LHV or between the MHV and RHV (if the MHV must be taken with segment 4 to improve the exposure and facilitate the dissection). Similarly to the right hepatectomy, venous confluences to the MHV are secured and divided with clips. Using the assistance of the Goldfinger, and after exposure of the MHV and/ or the LHV, we can pull the parenchyma toward the surgical as- 
Fig. 1. The Goldfinger dissector.
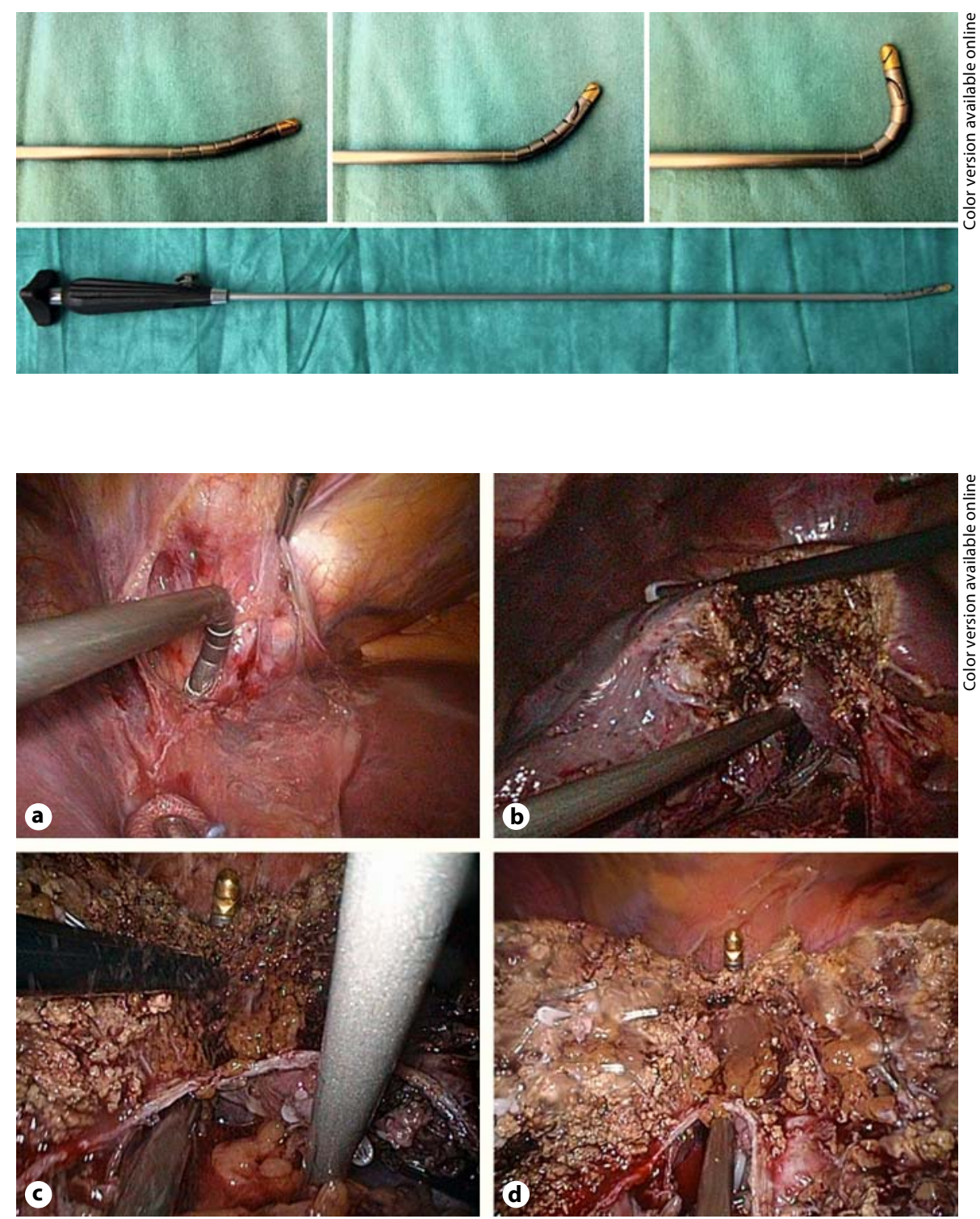

Fig. 2. a Upper dissection of the space between the RHV and MHV. b Infero-superior arcade through the space between the anterior surface of the vena cava and the posterior surface of the liver. c Lifting the liver cutting area from posterior to anterior direction during LLR. d Indication of parenchymal transection line.

pirator to complete the dissection and, finally, a second stapler is used to safely secure the veins.

\section{Results}

This modified hanging maneuver for LLR was utilized in 18 cases of right and left hepatectomy (table 1). The median patient age was 61 years (range 27.8-77.3). The female/male ratio was $61.1 / 38.9 \%$. Indications for liver resection were: colorectal metastases in 11 (61.1\%), liver cell adenoma in 5 (27.8\%), and breast metastases and hepatocellular carcinoma in 1 (5.5\%) case each.
The median resection time was 96 min (range 75-135), and the median blood loss was $220 \mathrm{ml}$ (range 180-1,250). No conversion was recorded. The preoperative diagnoses were confirmed at histological examination in all cases, showing a mean number of lesions of $1.8 \pm 1.4$ and a median size of total lesions of $11.3 \mathrm{~cm}$ (range 1.6-20). Multiple lesions were present in $6(33.3 \%)$ cases, all resulting in R0 resection. The median free margin was $4 \mathrm{~mm}$ (range $2-20$ ).

No perioperative mortality ( $\leq 3$ month) was recorded. During a median postoperative stay of 4.5 days (range $3-15)$, complications occurred in 2 (11.1\%) patients: an acute respiratory distress syndrome and a biliary leak spontaneously resolved. 
Table 1. Intraoperative data

\begin{tabular}{lc}
\hline Type of resection & \\
$\quad$ Right hepatectomy & $13(72.2)$ \\
$\quad$ Left hepatectomy & $5(27.8)$ \\
Median surgical time, min & $285(195-580)$ \\
Median anesthesia time, min & $480(255-720)$ \\
Median blood loss, ml & $220(180-1,250)$ \\
Specimen extraction & \\
$\quad$ Pfannenstiel incision & $15(75)$ \\
Previous incision & $3(15)$ \\
Median postoperative stay, days & $4.5(3-15)$ \\
\hline
\end{tabular}

$\mathrm{n}=18$. Figures in parentheses are percentages or ranges.

\section{Discussion}

From the pioneering era of LLR activity, tremendous progress has been made in terms of surgical technique and perioperative care of liver diseases.

LLRs are more difficult and require a longer learning curve compared to the standard approach to liver resection. It is obvious to think that mastering laparoscopic techniques together with an in-depth knowledge of liver surgery are both a prerequisite to subsequently develop LLR. There are several advantages offered by this technique; basically, overall reduced morbidity and postoperative pain, a shorter hospital stay and a fast recovery due to the limited trauma of the abdominal wall $[8,9]$. The described techniques in LLR range from the patient position to the number of trocars used, from the type of access (pure laparoscopic, hand-assisted technique, hybrid technique) to the techniques to divide the liver parenchyma and to prevent bleeding. Whereas the intraoperative results of LLR are the same as when using the standard approach in a selected patient population, the surgical technique of LLR is substantially different [8]. Several aspects in laparoscopy are different to the open approach: there is no tridimensional vision, there is a lack of tactile feeling, ultrasound evaluation is more difficult as is appreciation of the tumor margins, liver mobilization is less manageable, and the use of sutures is limited while the use of vascular staplers is almost mandatory. Another important difference between the standard and laparoscopic approach that applies to right or left hepatectomy is the direction or the angle of the transection line: antero-posterior in open surgery (or $90^{\circ}$ angle) compared to infero-superior $\left(30^{\circ}-45^{\circ}\right.$ angle) in LLR.

Belghiti's hanging maneuver was conceived for right hepatectomy with the open approach by pulling up the liver parenchyma facilitating exposure, dissection and coagulation of the intrahepatic structures. Originally, a tape was passed with a linear vascular clamp through the avascular space between the liver and the anterior wall of the IVC, escaping between the RHV and the MHV. This maneuver made it possible to obtain a wide opening of the two sides of the liver cutting surface, compressing the parenchyma from behind while also acting as a hemostatic tool.

The only contraindication for the hanging maneuver is neoplastic infiltration through the retrohepatic avascular space into the vena cava. Various modifications of this technique have been reported $[10,11]$. A nasogastric tube or a Penrose soft drain is sometimes used but tapes are usually preferred $[10,11]$. Others proposed a modified hanging maneuver for laparoscopic right hepatectomy in order to improve the exposure of the liver while providing a guide to the plane of parenchymal dissection [12]. They utilized a vascular tape that is ligated and retracted after mobilizing the right lobe from the diaphragm and before starting the resection. This technique was also described in hand-assisted LLRs [13].

However, we do think that this method may hamper the use of transection devices hindered by the vertical part of the tape and because the distribution of tape-induced pressure is not homogenous along the interior surface of the cutting line. To improve these limitations, we propose the use of the Goldfinger dissector at different stages of the procedure: (a) to dissect the space between the RHV and MHV; (b) to create the caudal-cranial arcade through the space between the anterior surface of the IVC and the posterior surface of the liver; (c) to lift the cutting area from the posterior to the anterior direction; (d) to indicate the transection line, and finally (e) to lift the major hepatic veins, facilitating the transection with vascular staplers.

Upward pressure of the Goldfinger provides a direct linear plane during parenchymal division and does not impede the correct use of dissector devices while facilitating the view and the exposure of the liver tissue and vascular structures.

Alternatively, the Goldfinger can be used to capture and pass a tape through the retrohepatic space. Unlike the original hanging maneuver, we use it only after initial dissection of the liver from the retrohepatic vena cava and after starting one third of the parenchyma division. Indeed, splitting a part of the liver first facilitates the exposure, and the pressure promoted by the Goldfinger is inversely proportional to the amount of the remnant parenchyma. 
We consider the Goldfinger an ideal instrument for the modified hanging maneuver in LLR due to its atraumatic tip with an angled shape, the optimal length and the strong consistency of the metallic material able to support the whole liver. Positioning of this tool does not extend the operation time but attention should be paid when making the dissection, avoiding any forced movement of the tip when resistance is encountered. The only problem could be represented by the need to put it in the trocar camera and to displace the optical device more laterally (which is not the case when using the tape).

In conclusion, the presented modified hanging maneuver for laparoscopic right and left hepatectomy based on the use of Goldfinger is safe, effective, and reproducible, and can be considered as an additional tool to facilitate major resections in LLR.

\section{References}

$>1$ Edwin B, Nordin A, Kazaryan AM: Laparoscopic liver surgery: new frontiers. Scand J Surg 2011;100:54-65.

$\checkmark 2$ Descottes B, Glineur D, Lachachi F, Valleix D, Paineau J, Hamy A, et al: Laparoscopic liver resection of benign liver tumors. Surg Endosc 2003; 17:23-30.

$>3$ Castaing D, Vibert E, Ricca L, Azoulay D, Adam R, Gayet B: Oncologic results of laparoscopic versus open hepatectomy for colorectal liver metastases in two specialized centers. Ann Surg 2009;250:849-855.

$>4$ Belghiti J, Guevara OA, Noun R, Saldinger PF, Kianmanesh R: Liver hanging maneuver: a safe approach to right hepatectomy without liver mobilization. J Am Coll Surg 2001;193: 109-111.
5 Ceelen W, Walder J, Cardon A, Van Renterghem K, Hesse U, El Malt M, et al: Surgical treatment of severe obesity with a low-pressure adjustable gastric band: experimental data and clinical results in 625 patients. Ann Surg 2003;237:10-16.

6 Saif R, Jacob M, Robinson S, Sen G, Manas D, White S: Laparoscopic Pringle's manoeuvre for liver resection?: How I do it. Minim Invasive Ther Allied Technol 2011.

7 Robinson SM, White SA: The use of Goldfinger (TM) (Ethicon Endo Surgery) to facilitate laparoscopic distal pancreatectomy. Ann R Coll Surg Engl 2009;91:524-525.

-8 Nguyen KT, Marsh JW, Tsung A, Steel JJ, Gamblin TC, Geller DA: Comparative benefits of laparoscopic vs open hepatic resection: a critical appraisal. Arch Surg 2011;146: 348-356.

$>9$ Vigano L, Laurent A, Tayar C, Tomatis M, Ponti A, Cherqui D: The learning curve in laparoscopic liver resection: improved feasibility and reproducibility. Ann Surg 2009; 250:772-782.
10 Utsunomiya T, Shimada M: Modified hanging method for liver resection. J Hepatobiliary Pancreat Sci 2012;19:19-24.

11 Suh KS, Lee HJ, Kim SH, Kim SB, Lee KU: Hangingmaneuverinlefthepatectomy.Hepatogastroenterology 2004;51:1464-1466.

12 Gayet B, Cavaliere D, Vibert E, Perniceni T, Levard H, Denet C, et al: Totally laparoscopic right hepatectomy. Am J Surg 2007;194: 685-689.

13 Koffron AJ, Auffenberg G, Kung R, Abecassis $\mathrm{M}$ : Evaluation of 300 minimally invasive liver resections at a single institution: less is more. Ann Surg 2007;246:385-392. 